\title{
Microgrid Modeling and Fuel Savings Opportunities Through Centralized Load Control
}

\author{
Spencer C. Shabshab, J. Kendall Nowocin, Peter Lindahl, and Steven B. Leeb
}

\begin{abstract}
Small microgrids can derive their electrical power from a variety of energy resources. Some of these, including U.S. Military Forward Operating Bases (FOBs), use diesel generators as the primary or sole resource. In almost all cases, efficient utilization of generation resources is a high priority. This is particularly so for FOBs, for which diesel fuel resupplies come at remarkable monetary, logistical, and safety costs. Increasing the fuel efficiency of such microgrids requires not only incremental improvements to generation and load services, but also a higherlevel understanding of how these components interact. This study of a typical U.S. Army FOB characterizes its power system, which is powered by diesel generators and has a load profile dominated by distributed environmental control units (ECUs). The study contributes an actionable simulation model of this power system and uses it to identify an opportunity for energy savings through appropriate scheduling of the ECUs.
\end{abstract}

\section{INTRODUCTION}

Small microgrids can derive their electrical power from a variety of energy resources. Some of these, including U.S. Military Forward Operating Bases (FOBs), operate in islanded modes and use diesel generators as a primary or sole energy resource. A primary goal for all such microgrids is to match energy generation to the demand of the grid's various service loads.

Various approaches to the problem of source-load balancing in microgrids are available. Generally, they employ a combination of generator dispatch [1], energy storage [2], and load management [3]. Each balancing tactic comprises a variety of implementations: generator dispatch can be reactive to information such as microgrid load or energy pricing, or it can be proactive based on some predictive optimization; Energy storage has a wide array of physical formats, ranging from pumped hydro storage to galvanic cells; Load managementthe manipulation of loads to shape the grid demand profilecan be enacted through economic incentives, enforced simply by "shedding" noncritical loads, or executed more carefully by selecting individual loads to delay or shed based on a cost function. Each of these three balancing tactics also has limitations: generator dispatch is ideal in that it adjusts generation capacity to match load demand, but it cannot effectively respond to fast load variations; Energy storage has a comparatively fast response time, but remains costly to implement; Demand response shapes the load profile such that it can be more easily serviced by available energy resources, but must be limited so that disturbances to load service remain within acceptable boundaries. The most effective blend of these strategies depends on the realities of the microgrid in which they are deployed.
This study of a U.S. Army FOB examines generator underutilization, driven in large part by the large peak-to-average ratio of the facility's erratic load profile, as a primary cause of fuel waste in the military microgrid. The programmatic underutilization of generation resources in isolated microgrids is well-documented [4], as is its causal connection to large demand peak-to-average ratios [5]. Many approaches to this problem seek to accommodate the erratic load profile by smoothing the load with dedicated energy storage [7], or by shedding noncritical loads according to some priority order when the need to store or supply energy exceeds the capacity of available storage resources [6]. In systems where energy storage is limited or unavailable, load shedding to smooth the demand profile ceases to be an emergency intervention and begins to become a part of normal operation. In this case, a load management strategy that takes into account the needs and flexibilities of the microgrid's key loads, as well as the capacity and dispatch speed of available generation resources, is needed to secure adequate load service and source-load balance.

In this study, we characterize and model the key loads (ECUs) and generation resources of an Army FOB based on field observations. We combine these models into an FOB simulation environment to investigate excessive fuel use caused by a base's high peak-to-average load ratio. Unpredictable and unnecessary "stacking" (i.e. simultaneous operation) of thermostatically controlled environmental control units (ECUs), can take a generator from underloaded to heavily loaded or overloaded in seconds. The stacking does not reflect a sudden increase in the power needed for environmental control, but rather the chance overlap of individual heater "on" periods. Energy storage can help to eliminate this problem, as achieved in [7], but is not always economically or operationally feasible. We propose a load management approach that leverages the inherent storage capacity of FOB tents to impose peak load constraints and continually eliminate the load stacking behavior on the minutes timescale while still meeting comfort requirements.

Section II introduces the FOB being observed in this study, describes its operation, and develops working models of its key components. Section III identifies the costly load stacking behavior, estimates its fuel cost using the actionable simulation developed in Section II, and demonstrates one example of how a centralized control scheme could eliminate unnecessary demand peaks and save fuel at little cost to thermal performance. Section IV summarizes the observations of this study and outlines the direction of future work. 


\section{SySTEM CHARACTERIZATION}

This study is based on observations of the Base Camp Integration Laboratory (BCIL) in Ft. Devens, MA. The BCIL is an archetypal FOB used to test new technologies for potential deployment to FOBs around the world. It contains the same generation resources, service loads, and structures typical of such facilities. A picture of the BCIL is included in Figure 1 to show the scale of the facility. The FOB energy demand is dominated by environmental control units, service loads which maintain the camp's various tents at acceptable temperatures. As shown in Figure 2, during heating operation the environmental control load accounts for over $75 \%$ of the total base load [8].

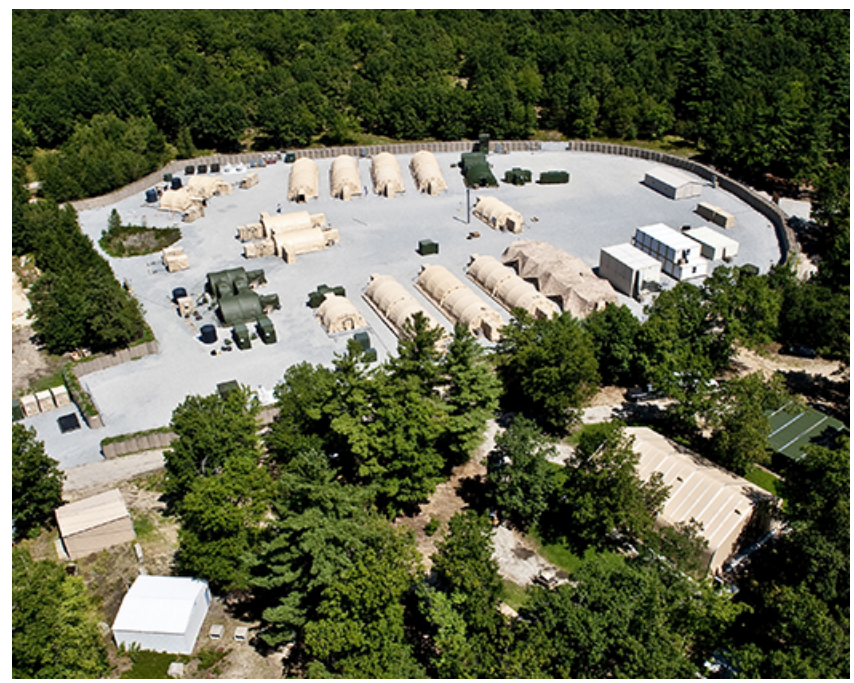

Fig. 1. Aerial photograph of the BCIL

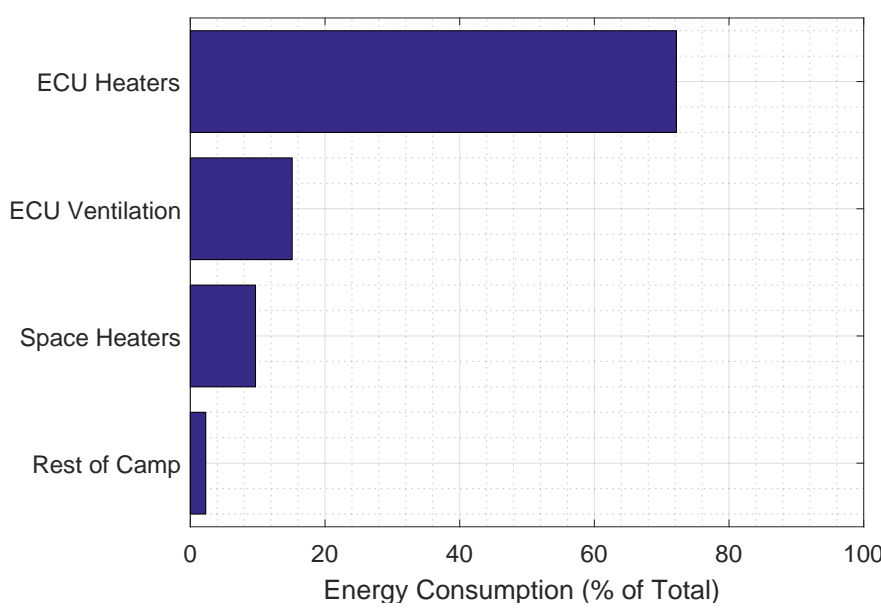

Fig. 2. A typical FOB load breakdown. This load profile was captured at the BCIL by the Deployable Nonintrusive Load Monitor (DepNILM) during 48 hours of occupancy by 90 soldiers [8].

A bank of parallel-connected diesel generators powers the BCIL in an islanded microgrid configuration. The generators automatically dispatch to keep the load within $30 \%$ and $80 \%$ of the total generation capacity. The purpose of this behavior is to appropriately load the active generators while also protecting them from overload in the event of sudden demand increases.

A major portion of the generator bank power output is used for environmental control of the base's various tents, most of which are berthing complexes for soldiers. A berthing complex is comprised of two adjacent sections, each of which has a thermal time constant on the order of 10 minutes and is temperature controlled individually by an ECU. During heating operation, the ECUs are on-off controlled to maintain the tent's air temperature within a predetermined band. This is a thermostatic control regime, in which changes to the heater operating state depend only on measured tent temperature and current heater state.

\section{A. Generator Characteristics}

1) Sizing and Configuration: The BCIL generators are $60 \mathrm{~kW}$ synchronous diesel generators directly connected to the microgrid through an interruption contactor. They are connected to one another in parallel, and can turn on and off according to their automatic dispatch rules.

2) Automatic Dispatch Rules: At least one generator is dispatched at all times. When load demand exceeds $80 \%$ of the total dispatched generation capacity for longer than 10 seconds, an additional generator spins up and comes online. When load demand decreases below $30 \%$ of the dispatched generation capacity for longer than approximately five minutes, one generator is taken offline and spins down.

3) Generator Model: For the purposes of this investigation, as in [7], the diesel generators are treated as ideal electrical sources and their fuel consumption is approximated by a slopeintercept equation as in the HOMER power system simulation software [9]. The fuel consumption rate of a single generator is approximated as,

$$
\dot{m}=\dot{m}_{o}+\dot{m}_{1} \frac{P_{\text {load }}}{P_{\text {rated }}}
$$

where $\dot{m}$ is the fuel rate of the generator bank $(\mathrm{kg} / \mathrm{s}), \dot{m}_{o}$ is the no-load fuel rate of a single generator, $\dot{m}_{1}$ is the slope at which a single generator's fuel rate increases as the normalized load moves from $0-100 \%, P_{\text {rated }}$ is the rated power of a single generator, and $P_{\text {load }}$ is the load supplied by the generator.

The diesel generators that power the BCIL are all of the same construction and rating, and when paralleled they share the load equally. (1) can therefore be expanded to describe the fuel consumption rate of multiple paralleled generators as shown in (2):

$$
\begin{aligned}
\dot{m} & =\left(\dot{m}_{o}+\dot{m}_{1} \frac{P_{\text {load }} / N}{P_{\text {rated }}}\right) N \\
& =\dot{m}_{o} N+\dot{m}_{1} \frac{P_{\text {load }}}{P_{\text {rated }}}
\end{aligned}
$$

where $N$ generators, each rated for $P_{\text {rated }}$, are providing a $\frac{1}{N}$ fraction of the total load $P_{\text {load }}$ and each one consumes fuel according to (1). The efficiency of $N$ generators supplying a total power of $P_{\text {load }}$ can be computed with (3):

$$
\eta=\frac{P_{\text {loadt } 1}}{\dot{m} L H V}
$$


where $P_{\text {load }}$ is the total electrical power provided by the generators $(\mathrm{kW}), \dot{m}$ is the total fuel rate of the generators ( $\mathrm{kg} / \mathrm{s})$ according to (2), and $L H V$ is the lower heating value of the generator fuel $(\mathrm{kJ} / \mathrm{kg})$ [9]. Variables $\dot{m}_{o}$ and $\dot{m}_{1}$ are approximated with a linear fit to typical fuel rate data points for a $60 \mathrm{~kW}$ diesel generator [10]. The resulting fuel rates and efficiencies of 1,2, and 3 generators providing power along their entire operating range, as well as the values calculated from the data points of [10], are shown in Figure 3.
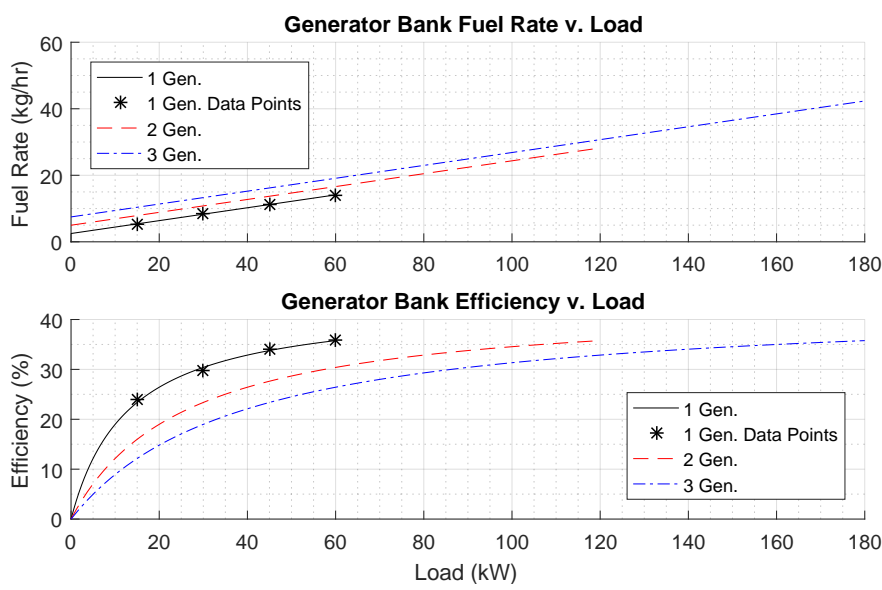

Fig. 3. Approximate fuel rate and efficiency curves for 1, 2, and 3 parallelconnected $60 \mathrm{~kW}$ diesel generators.

A bank of diesel generators modeled in this way and controlled according to the logic described in Section II-A2, gives a reasonable approximation of the diesel generators at the BCIL.

\section{B. Tent Characteristics}

1) Sizing and Configuration: The BCIL infrastructure consists primarily of berthing, amenity, and storage compartments. A large portion of these compartments are berthing complexes, in which soldiers sleep. The berthing complexes, which are semi-cylindrical in shape, have a footprint of approximately $75 \mathrm{ft}$. x $25 \mathrm{ft}$. (22.86 m x $7.62 \mathrm{~m})$. A complex is divided into two sections, and each section is serviced by a dedicated ECU.

2) ECU: An F100-60K ECU is connected to each tent section by one supply and one return air duct. The ECU can operate in either heating or cooling mode. In cooling mode, it rejects heat from the tent using a heat pump. In heating mode, the ECU operates a nominal 10kW resistive heater (field observations revealed power draws closer to $9 \mathrm{~kW}$ ), under a thermostatic regime. The thermostatic control logic and its performance are shown in Figure 4.

3) Thermal Characteristics: The berthing complexes are lined with an insulating layer to reduce heat loss to the environment. The two sections that make up each complex are connected by a passageway in the middle of the complex, which is a path for heat flow between tent sections.

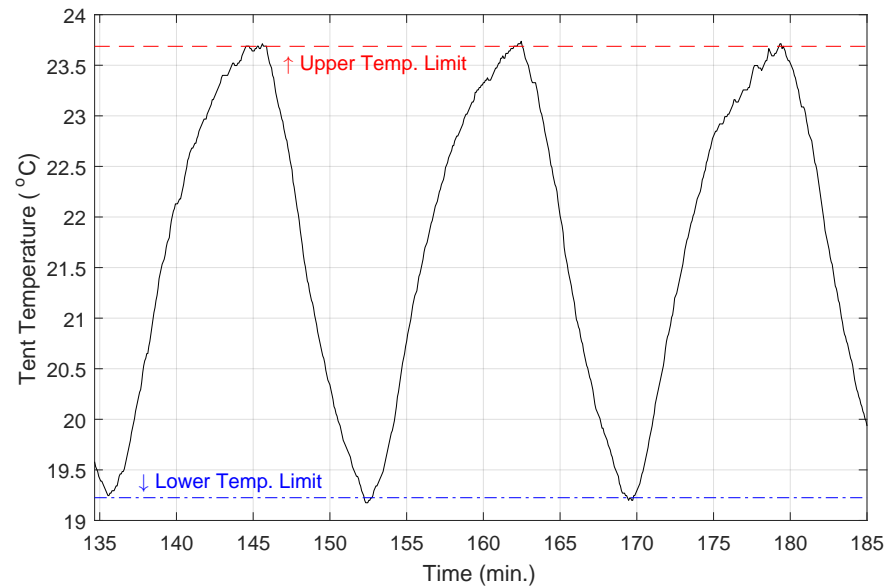

Fig. 4. Thermostatic ECU control regime.

4) Tent/ECU Model: A lumped parameter model of the berthing complex is depicted in Figure 5. Each tent section is modeled as a lumped thermal capacitance $\left(C_{t}\right)$ connected to a thermal reservoir representing the outdoor environment, through a constant lumped thermal impedance $\left(R_{t e}\right)$. The thermal capacitance of each tent section depends on its contents, which may include tables, desks, bunks, equipment, and a varying number of occupants. The thermal impedance between each tent section and the environment depends, among other things, on the quality of the seal, whether doors and windows are opened or closed, and the wind speed on the outer surface of the structure. In addition to the conductive heat loss from each tent section to the environment, there is also a path for convective heat flow between connected tent sections $\left(R_{t t}\right)$. An ECU, treated as a heat source, provides the control input to the thermal system according to its thermostatic control logic. To account for the fact that hot air must propagate from the ECU output through a duct and into the tent section before any heating actually occurs, a time constant is assigned to the ECU heat input. The solar flux and ECU heat inputs to each

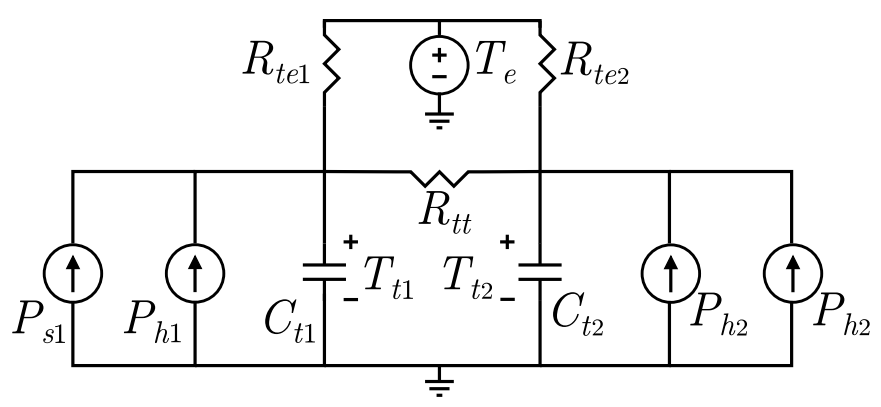

Fig. 5. Berthing complex model. Subscripts ' 1 ' refer to tent section 1, subscripts ' 2 ' refer to tent section 2 . 
tent section are further described by (4)-(5),

$$
\begin{aligned}
\dot{P}_{h} & =\alpha\left(P_{\text {nom }}-P_{h}\right) \\
P_{s} & =k P_{\text {sol }}
\end{aligned}
$$

where $\alpha$ is the time constant of the ECU heater input, $P_{\text {nom }}$ is the nominal output power of the heater, $P_{\text {sol }}$ is the solar irradiance measured at a central point of the $\mathrm{FOB}$, and $k$ is a scaling constant that accounts for the location and orientation of each tent section relative to the sun. In general, $k$ has a stochastic component due to atmospheric conditions and a deterministically time-variant component due to the trajectory of the sun and layout of the facility.

Four tent complexes comprising eight tent sections were modeled according to Figure 5 using a nonlinear least-squares estimation algorithm which, given a known series of system inputs, searches for model parameters that minimize the error between model response and measured response. Recorded environmental temperature, solar irradiance, heater states, and tent section temperatures from a test on May 22nd, 2018 are used here to fit the thermal model of Figure 5 to each tent complex. The result of this algorithm for one tent section is shown in Figure 6. The parameter values produced for all tent complexes are shown in Table I. In all cases, the average of the model response temperature was within $0.36^{\circ} \mathrm{F}\left(0.2^{\circ} \mathrm{C}\right)$ of the measured average. The model response predicted the measured maximum and minimum temperatures to within $1.26^{\circ} \mathrm{F}\left(0.7^{\circ} \mathrm{C}\right)$, and was at all times within $2.8^{\circ} \mathrm{F}\left(1.56^{\circ} \mathrm{C}\right)$ of the measured temperature. To further corroborate the fit, the model parameters fit to data from between 1600 and 2200 on May 22nd were used to predict all section temperatures from 2200 on May 22nd to 0400 on May 23rd. In this case the average temperature was estimated to within $0.77^{\circ} \mathrm{F}\left(0.43^{\circ} \mathrm{C}\right)$, the maximum and minimum to within $1.57^{\circ} \mathrm{F}\left(0.875^{\circ} \mathrm{C}\right)$, and at all times to within $2.8^{\circ} \mathrm{F}\left(1.56^{\circ} \mathrm{C}\right)$.

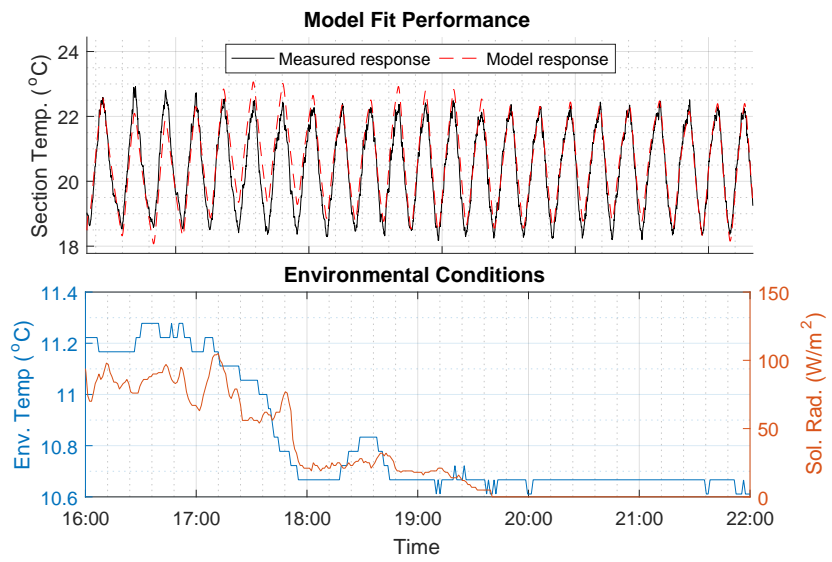

Fig. 6. Representative example of model fit performance for a single tent section.

It is worth noting that the model also replicates an important field observation: the ECUs under thermostatic control switch on and off at slightly different frequencies and as a result drift slowly in and out of phase with one another.

\begin{tabular}{|l|l|l|l|l|l|}
\hline Complex:Section & $C_{t}\left(\frac{k J}{K}\right)$ & $R_{t e}\left(\frac{K}{k W}\right)$ & $R_{t t}\left(\frac{K}{k W}\right)$ & $k_{s}$ & $\alpha$ \\
\hline $1: 1$ & 404.42 & 1.76 & 2.88 & 14.18 & 0.018 \\
\hline $1: 2$ & 387.46 & 2.27 & 2.88 & 9.82 & 0.019 \\
\hline $2: 1$ & 346.44 & 2.23 & 3.96 & 12.50 & 0.023 \\
\hline $2: 2$ & 367.98 & 1.81 & 3.96 & 11.70 & 0.016 \\
\hline $3: 1$ & 436.90 & 2.11 & 2.37 & 19.00 & 0.011 \\
\hline $3: 2$ & 332.42 & 1.90 & 2.37 & 16.10 & 0.012 \\
\hline $4: 1$ & 422.13 & 1.86 & 2.95 & 22.10 & 0.011 \\
\hline $4: 2$ & 444.12 & 1.92 & 2.95 & 15.80 & 0.013 \\
\hline
\end{tabular}

BERTHING COMPLEX MODEL PARAMETERS

\section{Fuel SAVINGS OPPORTUNITY}

Because the ECUs are thermostatically controlled, and further because they do not all cycle at exactly the same frequency, they must be expected to occasionally align in phase and demand power at the same time. This can generate demand peaks that do not correspond to the average power actually required for environmental control but rather to the chance coincidence of heater on periods. This behavior significantly increases the load profile peak-to-average ratio and can incur a considerable fuel cost. In what follows, simulation of base camp operation under both the traditional thermostatic ECU control scheme and under a centralized control scheme that prevents ECU stacking identifies the opportunity for fuel savings through central ECU scheduling.

\section{A. Simulation Testbed}

An object-oriented MATLAB simulation testbed, which includes the generator and thermal plant models described in this study, enables exploration of the costs and benefits associated with changing the ECU control scheme. It can simulate operation of the base camp with custom ECU control schemes under arbitrary, time-varying ambient temperature and solar irradiation conditions. Number of tent complexes, rating of generators, and initial number of generators operating can also be customized.

\section{B. Simulation Parameters}

The simulation in this study concerns four berthing complexes comprising eight tent sections, each section heated to a temperature setpoint of $68^{\circ} \mathrm{F}\left(20^{\circ} \mathrm{C}\right)$ by an ECU. The maximum aggregate load that the ECUs can produce is $72 \mathrm{~kW}$. A constant base load of $11 \mathrm{~kW}$ is assumed to account for ventilation (approximately $1 \mathrm{~kW}$ per ECU), lighting, and miscellaneous small loads. The ECUs are powered by a bank of two diesel generators, each rated for $60 \mathrm{~kW}$ and operated according to the logic described in Section II-A2. 
The environmental inputs, ambient temperature and solar irradiation, are taken from the measurements of a local weather station on May 22nd and May 23rd. The temperature varied between $51.1^{\circ} \mathrm{F}\left(10.6^{\circ} \mathrm{C}\right)$ and $52.3^{\circ} \mathrm{F}\left(11.3^{\circ} \mathrm{C}\right)$ during the observed period, from 1600 on May 22nd to 0400 on May 23rd. In each simulation, the initial turn-on time of each ECU is a uniformly distributed random variable between zero and 30 minutes.

\section{Simulation Under Thermostatic ECU Control}

The ECUs operate according to their thermostatic control law, turning on when the tent temperature reaches the lower bound of the comfort region and turning off when the tent temperature reaches the upper bound. All tent sections oscillate between the same temperature bounds, but at different frequencies due to differences in their thermal time constants. The fuel consumptions generated in five simulation runs average to $367.7 \mathrm{lbs}$. (166.8 $\mathrm{kg}$ ) or approximately $56.8 \mathrm{gal}$. (215.3 L) of JP-8 fuel. The results of one of the five simulation runs is shown for reference in Figure 7. The top plot shows how many heaters are operating. The middle plot shows, at every instance, the temperature of the hottest tent section and the temperature of the coolest tent section (all tent section temperatures are within the two lines at all times). The bottom plot indicates how many generators are operating. A second generator dispatches the first time the ECU load exceeds $48 \mathrm{~kW}$, in accordance with the automatic dispatch rules. Intermittent demand peaks in excess of $36 \mathrm{~kW}$ (the generator turn-off threshold), keep the second generator running almost continuously throughout the night.
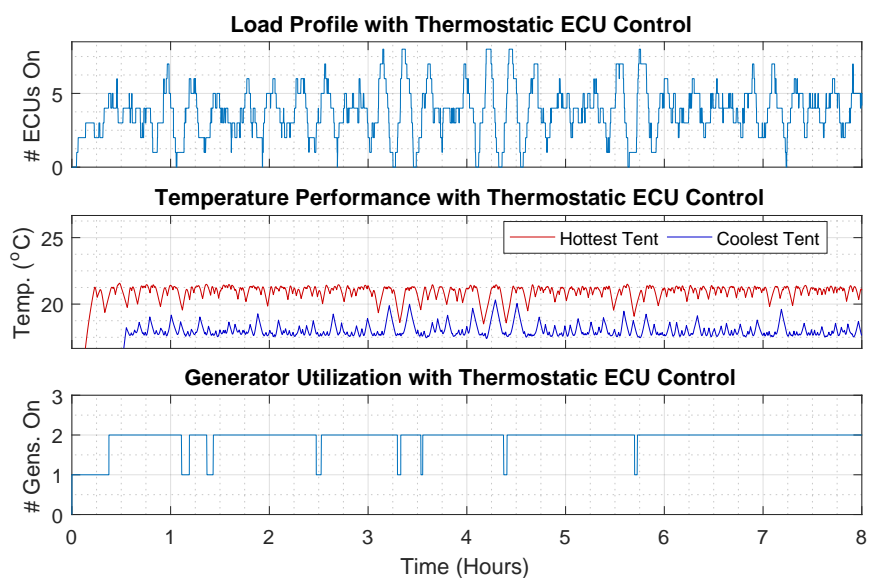

Fig. 7. Load profile, temperature performance, and number of generators active under thermostatic ECU control regime.

\section{Simulation Under Centralized Control}

1) Alternative Control Regime: As an alternative to the thermostatic ECU control approach, which has been shown to generate large peak loads and waste fuel, consider a centralized control scheme in which heater run times are scheduled on the minutes timescale such that peak load is minimized. The central controller, a block-diagram of which is shown in
Figure 8, uses environmental temperature $\left(T_{e}\right)$, expected solar irradiance $\left(P_{s}\right)$, the temperature setpoints $\left(T_{s e t}\right)$, and its model of each tent section to determine what average powers $\left(P_{\text {avg }}\right)$ are required. Temperature feedback of average tent section temperatures $\left(T_{t}\right)$ corrects for small model errors so that all tent section average temperatures converge to their setpoints in steady-state. A scheduler allots time slots to each ECU such that the average power each delivers equals its recommended control effort.

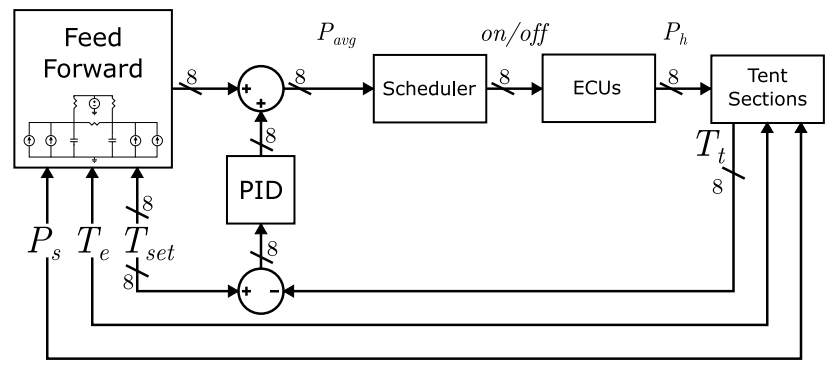

Fig. 8. Block diagram of the centralized control scheme. Lines labeled with an " 8 " indicate a signal bus containing a value for each of the eight tent sections simulated.

2) Centralized Control Performance: The control scheme applied above is now applied in simulation to the same eight tents under the same weather conditions. Model error with a statistical variance of $10 \%$ from the actual model parameters is simulated in the feed-forward controller. Five simulations under this centralized control scheme demonstrate that fuel consumption can be considerably decreased to $307 \mathrm{lbs}$. (139 $\mathrm{kg}$ ) or approximately 47.5 gal. (180 L) of JP-8 fuel- a fuel savings of $16.7 \%$. The performance of the centralized heater control scheme is shown in Figure 9. Note, in the top plot

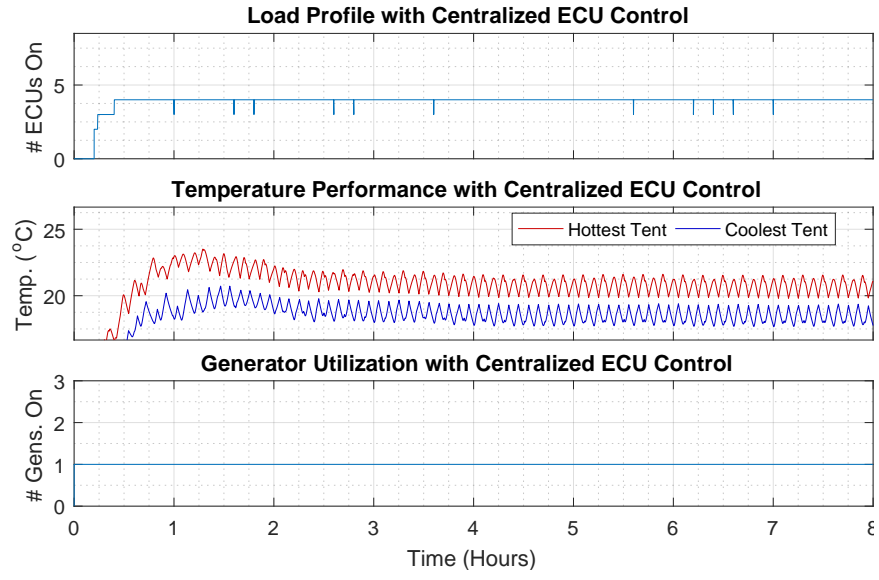

Fig. 9. Load profile, temperature performance, and number of generators active under synchronous ECU control regime.

of Figure 9, that the peak-to-average ratio of the ECU load profile is markedly improved. The middle plot shows that, with the exception of initial temperature overshoot due to model error and the integral temperature feedback, the temperature 
envelope of the tents is largely unchanged. In this simulation, the central controller allocated time slots such that two groups of four ECUs alternated being on and off. The middle plot of Figure 9 contains more ripple because of this grouping, but it should be noted that the maximum and minimum temperatures are approximately the same. Because the time constants of the tent sections are all slightly different, conversion to a synchronous control basis means that each tent sees a slightly different maximum and minimum temperature.

It should also be noted that the case simulated here is a particularly dramatic one, where the central controller is able to align heater time slots times perfectly such that four heaters are on at almost all times. Independently controlled heaters certainly cannot be expected to achieve such fine alignment in time, and so under thermostatic control they generate larger demand peaks frequently enough to keep the second generator running almost continuously. In all cases, however, a centralized control regime can guarantee a minimal peak-to-average ratio and the traditional thermostatic regime cannot.

A centralized control scheme could also do much more than limit demand peaks. Given a model such as the one presented here, a central controller could estimate what temperature performance can be achieved with a certain generation capacity. Alternatively, given temperature specifications, it could provide advance notification of what generation capacity will be required. This control scheme will be developed further in future research, as will controller hardware for field testing.

\section{CONCLUSION}

Theoretically, dedicated energy storage of sufficient storage and output capacity will always be able to absorb load fluctuations of any size and enable generators to run in a more optimally-loaded state. In practice, it is sometimes more economical to prevent load fluctuations altogether through load management. In the case of ECU stacking explored in this study, the management could be as simple as controlling the phase of each ECU's heater on/off cycle such that overlap is minimized. During slow-changing environmental conditions, load management of this kind can be achieved at little or no cost to temperature performance.

\section{ACKNOWLEDGMENT}

The authors would like to thank the the Office of Naval Research NEPTUNE Program and the Grainger Foundation for their support. They would also like to thank the administrators and staff at the Base Camp Integration Laboratory (BCIL) for their expertise and support.

\section{REFERENCES}

[1] Y. Han, P. Young, and D. Zimmerle, "Constrained optimum generator dispatch for fuel consumption minimization," in 2013 IEEE Power Energy Society General Meeting, July 2013, pp. 1-5.

[2] S. X. Chen and H. B. Gooi, "Sizing of energy storage system for microgrids," in 2010 IEEE 11th International Conference on Probabilistic Methods Applied to Power Systems, June 2010, pp. 6-11.

[3] B. Moran, "Microgrid load management and control strategies," in 2016 IEEE/PES Transmission and Distribution Conference and Exposition ( $T$ D), May 2016, pp. 1-4.
[4] J. Andriullu, A. Gates, H. Haynes, L. Klett, S. Matthews, E. Nawrocki, P. Otaduy, M. Scudiere, T. Theiss, J. Thomas, L. Tolbert, M. Yauss, and C. Voltz;, "Advanced power generation systems for the 21st century: Market survey and recommendations for a design philosophy," Oak Ridge National Laboratory, Tech. Rep., 1999.

[5] Y. Bhandari, S. Chalise, J. Sternhagen, and R. Tonkoski, "Reducing fuel consumption in microgrids using pv, batteries, and generator cycling," in IEEE International Conference on Electro-Information Technology, EIT 2013, May 2013, pp. 1-4.

[6] F. Verrilli, G. Gambino, S. Srinivasan, G. Palmieri, C. D. Vecchio, and L. Glielmo, "Demand side management for heating controls in microgrids," 2016.

[7] R. L. Kelly, G. Oriti, and A. L. Julian, "Reducing fuel consumption in a forward operating base using an energy management system," in 2013 IEEE Energy Conversion Congress and Exposition, Sept 2013, pp. 1330-1336.

[8] M. Gillman, W. Singleton, R. Wilson, W. Cotta, J. Donnal, J. Paris, and S. Leeb, "Accounting for every kilowatt," Defense AT\&L, 2014. [Online]. Available: http://www.rle.mit.edu/esg/wp-content/uploads/201 6/08/GillmanNEW.pdf

[9] How homer creates the generator efficiency curve. HOMER. [Online] Available: https://www.homerenergy.com/products/pro/docs/3.11/how $\searrow_{-}$ homer $\backslash$ creates $\backslash$ _the $\backslash$ _generator $\backslash$ _efficiency $\backslash$ _curve.html

[10] Approximate diesel fuel consumption chart. Diesel Service and Supply. [Online]. Available: http://www.dieselserviceandsupply.com/Diesel $\backslash$ _F uel $\backslash \_$Consumption.aspx 